\title{
QT interval in anorexia nervosa
}

\author{
R A Cooke, J B Chambers, R Singh, G J Todd, N C Smeeton, J Treasure, T Treasure
}

\begin{abstract}
Objectives-To determine the incidence of a long QT interval as a marker for sudden death in patients with anorexia nervosa and to assess the effect of refeeding. To define a long QT interval by linear regression analysis and estimation of the upper limit of the confidence interval (95\% CI) and to compare this with the commonly used Bazett rate correction formula.
\end{abstract}

Design-Prospective case control study. Setting-Tertiary referral unit for eating disorders.

Subjects -41 consecutive patients with anorexia nervosa admitted over an 18 month period. 28 age and sex matched normal controls.

Main outcome measures-maximum QT interval measured on 12 lead electrocardiograms.

Results $-43.6 \%$ of the variability in the QT interval was explained by heart rate alone $(p<0.00001)$ and group analysis contributed a further $5.9 \%(p=0.004)$. In $6(15 \%)$ patients the QT interval was above the upper limit of the $95 \%$ CI for the prediction based on the control equation (NS). Two patients died suddenly; both had a QT interval at or above the upper limit of the $95 \%$ CI. In patients who reached their target weights the QT interval was significantly shorter (median 9.8 ms; $p=0.04$ ) relative to the upper limit of the $60 \% \mathrm{CI}$ of the control regression line, which best discriminated between patients and controls. The median Bazett rate corrected QT interval (QTc) in patients and controls was $435 v$ $405 \mathrm{~ms}^{-1 / 2}(p=0.0004)$, and before and after refeeding it was $435 v 432$ ms.s ${ }^{1 / 2}$ (NS). In 14(34\%) patients and three (11\%) controls the QTc was $>440{\mathrm{~ms} . \mathrm{s}^{-1 / 2}}^{(\mathrm{p}=}$ 0.053).

Conclusions-The QT interval was longer in patients with anorexia nervosa than in age and sex matched controls, and there was a significant tendency to reversion to normal after refeeding. The Bazett rate correction formula overestimated the number of patients with QT prolongation and also did not show an improvement with refeeding.

(Br Heart f 1994;72:69-73)

Anorexia nervosa affects about $1 \%$ of all women between 15 and $25 .{ }^{1}$ Men and other age groups are affected although much less commonly. The risk of death in long-term follow up studies (more than 4 years) is about $10 \% .^{2}$ A proportion die suddenly with no cause found at postmortem examination. ${ }^{34}$ It has been proposed that these deaths are a result of cardiac arrhythmias for which a long QT interval on the electrocardiogram is a marker. ${ }^{4}$ The evidence for a long QT interval is conflicting and based on a few studies of small numbers of patients. ${ }^{4-7}$ All have used the Bazett formula to correct for heart rate; this formula tends to overcorrect at high heart rates and undercorrect at low rates. ${ }^{8-11}$ Both bradycardia and tachycardia may be found in anorexia nervosa. ${ }^{1213}$ Moreover few studies have used internal controls and hence differences in method such as lead selection and measurement techniques may have introduced further errors. ${ }^{5-7}$

The aims of this study were (a) to compare the QT interval in a large unselected sample of patients with anorexia nervosa and normal controls, (b) to compare the QT interval in patients with anorexia nervosa before and after treatment, and (c) to compare the results with and without the Bazett rate correction formula.

\section{Patients and methods}

PATIENTS

Forty one consecutive patients admitted between July 1990 and January 1992 to the Maudsley Hospital for treatment of anorexia nervosa were studied. All were tertiary referrals to the specialist unit for eating disorders. The median (range) duration of illness was $9.7(0.5$ to 23.0$)$ years. Anorexia nervosa was defined by standard criteria according to the Diagnostic and Statistical Manual of Mental Disorders IIIR and the International Classification of Diseases, version 10 . Thirteen (32\%) had bulimia and $11(27 \%)$ were laxative abusers. The median (range) age was 26 ( 15 to 60$)$. Two (5\%) were men. Twenty eight asymptomatic age and sex matched controls were recruited from the nursing and medical staff of Guy's and the Maudsley Hospitals.

Serum electrolytes were measured on the same day as the electrocardiographic recording in all patients. The median (range) delay from admission was five ( 0 to 51 ) days. Echocardiography was performed. One patient had mitral valve prolapse. No patient or control were taking medication, and none had a family history of deafness or sudden death. 
Twenty three (56\%) patients reached their target weights and the QT interval was compared in this group before and after treatment.

\section{MEASUREMENT OF QT INTERVAL}

Twelve lead electrocardiograms were recorded at a paper speed of $50 \mathrm{~mm} / \mathrm{s}$ on a three channel Mingograph (Siemens, Sunbury on Thames) in the morning after the patient had rested in a semirecumbent posture for five minutes. All measurements were made with callipers by a single observer who was unaware of the patients' clinical characteristics. The QT interval was measured from the initial downward deflection of the QRS complex to the end of the $T$ wave determined by the criteria described by Lepeshkin and Surawicz. ${ }^{14}$ It was measured in every lead where the $T$ wave was visible. The $R R$ interval was calculated from the cycle preceding the one on which the maximum QT interval was measured.

\section{DATA ANALYSIS}

The maximum measured QT interval was used for analysis. This was plotted against the $R R$ interval for patients with anorexia nervosa and normal controls. The factors controlling variability of the measured QT intervals were investigated by multiple linear regression with models constructed from the $R R$ interval, age, and group. To determine the number of patients with a long QT interval, the upper limit of the $95 \%$ confidence interval $(95 \% \mathrm{CI}$, one tailed) for the prediction with the control regression equation was drawn.

Although the deviations from the control regression line were almost symmetrical, their distribution was more peaked than expected from a normal distribution. Thus a line of discrimination was drawn corresponding to the upper limit of the $60 \% \mathrm{CI}$ for the prediction based on an assumption of normality, which allocated $72 \%$ of cases to the correct group. The QT interval in patients before and after refeeding was compared in relation to this line.

The Bazett rate corrected QT interval (QTc) was calculated with the formula QTc = $\mathrm{QT} / \sqrt{\mathrm{RR}}$, where the QT interval is measured in $\mathrm{ms}$ and the $R R$ interval in $s .^{8}$ The group medians were compared, and to estimate the proportion of patients with a long QT interval a QTc >440 ms. $\mathrm{s}^{-1 / 2}$ was taken as an arbitrary although commonly used upper limit of normal. ${ }^{15}$

A comparison was made of the QT dispersion, calculated by subtracting the minimum from the maximum measured QT interval on

Table 1 Median (range) data from patients (before treatment) and controls

\begin{tabular}{lccl}
\hline & $\begin{array}{c}\text { Patients } \\
(n=42)\end{array}$ & $\begin{array}{l}\text { Controls } \\
(n=28)\end{array}$ & $p$ Value \\
\hline Age (yr) & $26(15-60)$ & $26(19-63)$ & NS \\
Female (\%) & $39(95 \%)$ & $26(93 \%)$ & NS \\
Weight (kg) & $36(28-46)$ & $55(45-100)$ & $<0 \cdot 00001$ \\
Maximum QT (ms) & $380(340-440)$ & $380(340-440)$ & NS \\
RR (ms) & $780(560-1100)$ & $860(620-1220)$ & $0 \cdot 03$ \\
QT dispersion (ms) & $40(20-80)$ & $40(20-60)$ & NS \\
\hline
\end{tabular}

the 12 lead electrocardiogram. The leads in which the maximum QT interval was recorded were also compared.

Descriptive data are given as either the median (range) or percentage. Continuous variables were compared with the MannWhitney $U$ test for unpaired, and the Wilcoxon signed rank test for paired samples. The $\chi^{2}$ analysis was used for comparison of proportions. A $p$ value of $<0.05$ was considered significant.

\section{Results}

MAXIMUM QT INTERVAL BEFORE TREATMENT None of the electrocardiograms showed long PR or QRS intervals. There were no $T$ wave abnormalities. The measured QT interval was the same $(380 \mathrm{~ms})$, and the $\mathrm{RR}$ interval shorter (780 ms $v 860 \mathrm{~ms}, \mathrm{p}=0.03)$ in patients with anorexia than in controls (table 1). Of the variance in the QT interval $43 \%$ was explained by the RR interval alone ( $p<$ 0.0001 ). A further $5.9 \%$ of the variance was explained by the patient group. This value, although small, was significant $(p=0.004)$. In six $(15 \%)$ patients and one $(4 \%)$ control the measured QT interval was above the upper limit of the $95 \% \mathrm{CI}$ of the prediction based on the control regression equation (NS, fig 1).

The line that discriminated optimally between patients and controls corresponded to the upper limit of the $60 \%$ CI based on an assumption of normality (fig 1). Significantly more patients, than controls $(29(71 \%) v$ $7(25 \%))$ had values above this line $(p=$ 0.005 ). The median (range) upward deviation from the line for patients was $10(-31$ to 69$)$ ms.

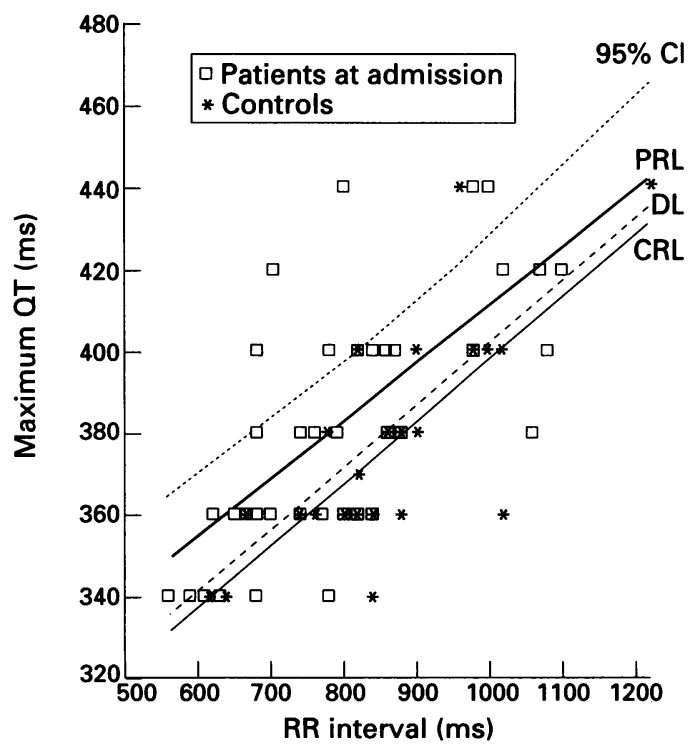

Figure 1 Plot of $Q T v R R$ interval for patients at admission and controls. The regression lines for patients (PRL; $Q T=270+0 \cdot 141 \times R R)$ and controls (CRL $Q T=246+0 \cdot 152 \times R R$ ) are shown. The number of patients with $Q T$ prolongation, the upper limit of the $95 \%$ CI for the prediction with the control regression equation and the line of optimal discrimination (DL; upper limit of the $60 \%$ CI based on assumption of normality) are shown. 
Table 2 Median (range) data from patients before and after refeeding

\begin{tabular}{lccl}
\hline & $\begin{array}{l}\text { Before } \\
(n=23)\end{array}$ & $\begin{array}{l}\text { After } \\
(n=23)\end{array}$ & $p$ Value \\
\hline Weight (kg) & $36(28 \cdot 1-45 \cdot 6)$ & $51(42 \cdot 6-67 \cdot 6)$ & $<0 \cdot 00001$ \\
Maximum QT (ms) & $380(340-440)$ & $360(320-420)$ & NS \\
RR (ms) & $780(610-1060)$ & $720(560-1160)$ & NS \\
QT dispersion (ms) & $40(20-80)$ & $40(20-60)$ & NS \\
\hline
\end{tabular}

Figure 2 Plot of $Q T$ v $R R$ interval before and after treatment for 23 patients with anorexia nervosa who reached their optimal weight. The line of discrimination $(D L)$ between patients and controls is drawn.



MAXIMUM QT INTERVAL AFTER TREATMENT Twenty three patients with anorexia were discharged after reaching their target weights. The median (range) time interval between admission and discharge was 88 (22 to 188$)$ days, and the median (range) weight gain was $14.2(7.6$ to $25 \cdot 7) \mathrm{kg}$. The measured QT interval decreased by $20 \mathrm{~ms}$ and the RR interval by $60 \mathrm{~ms}$ (NS, table 2 ).

There was a significant shortening of the QT interval in relation to the line of discrimination between patients and controls (median $9.8 \mathrm{~ms}$, Wilcoxon signed rank test, $\mathrm{p}=0.04$; fig 2 ). In four ( $17 \%$ ) patients before and two (9\%) after reaching their target weights the QT interval was above the upper limit of the $95 \% \mathrm{CI}$ for the prediction based on the control regression equation (NS); and in 15 $(65 \%)$ patients before and $14(61 \%)$ patients after reaching their target weights the QT interval lay above the line of discrimination (NS).

BAZETT RATE CORRECTION

The median QTc was significantly longer in patients (435 ms.s ${ }^{-1 / 2}$ ) than in controls (405

Table $3 Q T$ interval before and after refeeding in two patients who died suddenly

\begin{tabular}{|c|c|c|c|c|}
\hline & \multicolumn{2}{|c|}{ Patient 1} & \multicolumn{2}{|c|}{ Patient 2} \\
\hline & Before & After & Before & After \\
\hline $\begin{array}{l}\text { Weight (kg) } \\
\text { Maximum QT (ms) } \\
\text { RR (ms) } \\
\text { QT dispersion (ms) } \\
\text { Upper limit of } 95 \% \mathrm{CI}(\mathrm{ms}) \\
\text { QTc }\left(\mathrm{ms}^{-1 / 2}\right)\end{array}$ & $\begin{array}{r}42 \\
380 \\
680 \\
40 \\
380 \\
461\end{array}$ & $\begin{array}{r}67 \\
340 \\
580 \\
40 \\
366 \\
446\end{array}$ & $\begin{array}{r}33 \\
400 \\
780 \\
80 \\
395 \\
453\end{array}$ & $\begin{array}{r}55 \\
400 \\
860 \\
60 \\
406 \\
431\end{array}$ \\
\hline
\end{tabular}

ms.s $\left.\mathrm{s}^{-1 / 2}, \mathrm{p}=0.0004\right)$. The QTc before and after refeeding was $435 v 432 \mathrm{~ms} . \mathrm{s}^{-1 / 2}$ (NS). In $37(90 \%)$ patients and $20(71 \%)$ controls the QT interval was above the Bazett line (QTc > $400 \mathrm{~ms} . \mathrm{s}^{-1 / 2}$ (NS), and in 14 (34\%) patients and three $(11 \%)$ controls the QTc was $>440$ ms.s ${ }^{-1 / 2}(p=0.053)$.

QT DISPERSION AND LEAD DISTRIBUTION OF THE MAXIMUM MEASURED QT INTERVAL The lead showing the longest QT interval was most commonly lead V3 (82\%) in controls, and lead V2 (63\%) in patients with anorexia. There were no significant differences in QT dispersion between patients and controls, or between patients before and after refeeding (tables 1 and 2).

\section{CLINICAL CORRELATES}

Two patients died suddenly. In both cases this occurred after discharge from hospital and after a relapse. No arrhythmias were documented and there were no recorded electrocardiograms immediately before death. In both cases the QT interval was at or above the upper limit of the $95 \%$ CI before and became normal after refeeding (table 3 ).

No patient had hypocalcaemia or hypomagnesaemia based on our accepted laboratory definition of the normal range. In six (15\%) patients the serum potassium was $<3.5 \mathrm{mmol} / 1$ at admission; in two (33\%) of these the QT interval was above the $95 \% \mathrm{CI}$. Serum potassium was within the normal range (3.5 to $5.0 \mathrm{mmol} / \mathrm{l})$ in the rest of the patients, four $(11 \%)$ of whom had a QT interval above the $95 \%$ CI. Neither patient who died suddenly had a documented abnormal serum potassium.

\section{Discussion}

In our study we found a significantly longer QT interval in patients with anorexia nervosa than in age and sex matched controls. Previous studies of the QT interval in anorexia nervosa have been conflicting. This is in part explained by difficulties in comparison due to the wide variability of the $\mathrm{QT}$ interval with heart rate. Forty four per-cent of its variability was attributed to heart rate alone, and the separation into patient and control groups accounted for a further $6 \%$, a small but significant group difference.

PREVIOUS STUDIES AND THE BAZETT FORMULA The Bazett rate correction formula is commonly used to correct the QT interval for heart rate. This is a formula that is convenient and easy to use, but an approximation that may miss or exaggerate abnormalities. ${ }^{16}$ Moreover its use in previous studies of patients with anorexia nervosa has relied on historical controls to define normality. As measurement of the QT interval is highly dependent on the method used, in the absence of any agreed standard the failure to use internal controls is an important omission.

Thus Thurston and Marks reported a long QT interval in five out of nine patients with 
anorexia nervosa, which was unexplained by electrolyte imbalance or drugs. ${ }^{5}$ The upper limit of normal was defined by the Bazett line (QTc > $400 \mathrm{~ms}^{-1 / 2}$ ). If we had used this most patients and controls (37/41 (90\%) and 22/28 $(71 \%)$ ) would have had a long QT interval. A more commonly used definition of normal is a QTc $>440 \mathrm{~ms} . \mathrm{s}^{-1 / 2}$. Isner et al reported a long QT in three young patients with anorexia who died suddenly, two of whom had ventricular tachycardia and syncope within the preceding 24 hours. ${ }^{4}$ The QTc intervals were 460, 470, and $610 \mathrm{~ms} . \mathrm{s}^{-1 / 2}$. The numbers involved, however, amount to no more than a case series, and in at least one patient the length of the QT interval may have been explained by an electrolytic abnormality. By comparison, we

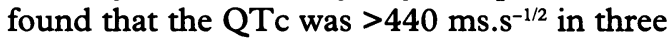
of our normal controls. Its use also overestimated the number of patients with a long QT interval compared with the upper limit of the $95 \%$ CI based on the control regression line $(14 / 41(34 \%) v 6 / 41(15 \%))$.

By contrast, Powers et al found no long QT intervals in 15 patients with anorexia nervosa either on admission or after reaching $75 \%$ of ideal body weight. ${ }^{6}$ The QTc range was 290 to $340 \mathrm{~ms} . \mathrm{s}^{-1 / 2}$. This is considerably lower than the range for healthy normal controls, and suggests either that there were inaccuracies due to the use of the Bazett formula or differences in method. Similarly, Gottdiener et al found no long QT intervals in 11 patients with anorexia nervosa, ${ }^{7}$ but an omission to measure the QT interval in any other lead than lead II may explain this finding. Thus we found that the maximum QT interval was most commonly measured in the anterior chest leads, which is in agreement with previous reports. ${ }^{17}$

The finding in our study that a long QT interval occurred in anorexia nervosa is consistent with reports of a long QT interval in healthy volunteers undergoing experimental starvation, ${ }^{18}$ in patients after gastroplasty or ileojejeunal bypass for morbid obesity, ${ }^{19-21}$ in dieters using the "liquid protein modified fast" diet, ${ }^{22}$ and in untreated coeliac disease. ${ }^{23}$

\section{HYPOTHESES FOR A LONG QT INTERVAL IN ANOREXIA NERVOSA}

The pathological basis of a long QT interval in anorexia nervosa is unknown. Cardiac muscle is lost in proportion to loss of body mass and there is no convincing evidence of impairment of ventricular function in these patients. ${ }^{6}$ Histological specimens have not shown myocarditis. ${ }^{6}$ Abnormalities of the hypothalamus are well described and increased autonomic tone has been suggested as a possible mechanism for lengthening the QT interval. ${ }^{512}$ In our study there were no incidences of noteable sinus bradycardia or conduction abnormalities. Autonomic function, was not studied.

\section{CLINICAL IMPLICATIONS}

The QT interval is a measure of myocardial repolarisation and its length is associated with life threatening ventricular tachycardias. Two patients died suddenly, both of whom had a measured QT interval at or beyond the upper limit of the $95 \%$ CI. In most patients, however, the increased length of the QT interval was small. The median increase above the line of discrimination between patients and controls was $10 \mathrm{~ms}$, and in only $10(24 \%)$ patients was the lengthening $>20 \mathrm{~ms}$. It is possible, however, that there is abnormal rate adaptation in these patients so that the QT interval would be longer at moments of stress or at low heart rates. This should be investigated in future studies.

For the recognition of patients who are at particular risk of sudden death measurement of the QT interval has a poor predictive value. A measured QT interval $>600 \mathrm{~ms}$ is associated with a significant risk of sudden death, ${ }^{24}$ but few patients will have such long QT intervals. The longest measured QT interval in our study was $440 \mathrm{~ms}$. An increased QT dispersion has been associated with sudden death in patients with a congenitally long QT interval but not in patients after myocardial infarction. ${ }^{25} 26$ The maximum QT dispersion in our study group was $80 \mathrm{~ms}$, which is considerably less than that found to be associated with torsade de pointes by Day et al. ${ }^{25}$

\section{EFFECT OF REFEEDING}

In patients who reached their target weights the median heart rate did not change significantly. But there was a significant shortening of the QT interval in relation to the line of discrimination between controls and patients before treatment. In $14(61 \%)$ of these patients the QT interval remained above the line of discrimination, and in two (9\%) it was above the limit of the $95 \%$ CIs. Thus there was not a complete reversion to normal. The Bazett formula showed only a small and not significant improvement in the QT interval after treatment.

\section{LIMITATIONS OF THE STUDY}

A long QT interval may be caused by electrolyte imbalance, notably hypocalcaemia ${ }^{27}$ and hypomagnesaemia. ${ }^{28}$ Serum calcium and magnesium were normal in all, and serum potassium was below the normal laboratory range in six patients. In two of these the QT interval was above the upper limit of the $95 \%$ CI. We used the method recommended by Lepeskekin and Surawicz to measure the QT interval. This avoids inclusion of the $U$ wave. ${ }^{14}$ As hypokalaemia may produce $U$ waves but not a long QT interval it is unlikely to account for the lengthened QT interval found in our study. We did not measure serum electrolytes in the control group and therefore cannot exclude the potentially confounding influence of minor electrolytic differences within the normal range.

Long QT intervals may also result from cardiomyopathy, ${ }^{29}$ severe myocardial ischaemia, or infarction, ${ }^{15} 30$ although not the presence of coronary stenoses alone. ${ }^{31}$ The probability of coronary artery disease in this population of patients is low, and no patient had electrocardiographic, symptomatic, or 
echocardiographic evidence of myocardial ischaemia or left ventricular dysfunction.

We did not use digitising techniques to measure the QT interval. All measurements were made by hand by a single observer who was unaware of the patient's group. Although we measured the QT dispersion it should be noted that the 12 leads of the electrocardiogram were recorded sequentially. It is not yet known whether minor beat to beat variations in the RR interval could affect QT dispersion.

In conclusion, until now the effect of anorexia nervosa on the QT interval has been uncertain. This study provides good evidence of long QT intervals with a significant tendency to reversion to normal after refeeding. An important substrate for life threatening arrhythmias and sudden death is quantified. The use of the Bazett formula, and also the omission of internal controls may explain previously conflicting reports.

We thank Professor Russell for permission to study his patients. JBC was supported by the Dowager Peel Medical pesearch Trust and the study was supported by a British Heart Foundation grant (BHF/7646)

1 Royal College of Psychiatrists. Eating disorders. London: Royal College of Psychiatrists, 1992. Council Report CR 14.

2 Fohlin L, Freyschuss U, Bjarke B, Davies CTM, Thoren C. Function and dimensions of the circulatory system in anorexia nervosa. Acta Paediatrica Scandinavica 1978, 67:11-6.

3 Bruch H. Death in anorexia nervosa. Psychosom Med 1971 33:135-44.

4 Isner JM, Roberts WC, Heymsfield SB, Yager J. Anorexia nervosa and sudden death. Ann Intern Med 1985;102: 49-52.

5 Thurston J, Marks P. Electrocardiographic abnormalities in patients with anorexia nervosa. Br Heart $\mathcal{F}$ 1974;36: in patien.

6 Powers PS, Schocken DD, Feld J, Holloway JD, Boyd F. Cardiac function during weight restoration in anorexia nervosa. International fournal of Eating Disorders 1991;10: n21-30.

7 Gottdiener JS, Gross HA, Henry WL, Borer JS, Ebert MH. Effects of self-induced starvation on cardiac size $\mathrm{MH}$. Effects of self-induced starvation on cardiac size and functio

8 Bazett HC. An analysis of the time relationships of electrocardiograms. Heart 1920;7:353.

9 Marion CV, Whitsett TL, Wilson MF. Applicability of correcting the QT interval for heart rate. Am Heart $f$ 1980;99:678.
10 Kovacs SJ. The duration of the QT interval as a function of heart rate: a derivation based on physical principles and a comparison to measured values. Am Heart $f$ and a comparisc

11 Campbell RWF, Gardiner P, Amos PA, Chadwick D, Jordan RS. Measurement of the QT interval. Eur Heart $\mathcal{f}$ 1985;6(suppl D):81

12 Palossy $B$, OO M. ECG alteration in anorexia nervosa. Adv Cardiol 1977;19:280-2.

13 Brotman AW, Stern TA. Case report of cardiovascular abnormalities in anorexia nervosa. Am f Psychiatry 1983 140:1227-8.

14 Lepeshkin E, Surawicz B. The measurement of the QT interval of the electrocardiogram. Circulation 1952;6: 378.

15 Schwartz PJ, Wolf S. QT interval prolongation as a predictor of sudden death in patients with myocardial infarction. Circulation 1978;57:1074-7.

16 Browne KF, Zipes DP, Heger Jj, Prystowsky EN. The influence of the autonomic system on the QT interval in man. Am $\mathcal{F}$ Cardiol 1982;50:1099-103.

17 Chambers JB, Ward DE. The QT and $\mathrm{QS}_{2}$ intervals in patients with mitral leaflet prolapse. Am Heart $\mathcal{f} 1987$ 114:355-61.

18 Simonson E, Henschell A, Keys A. The electrocardiogram of man in semistarvation and subsequent rehabilitation. Am Heart $\mathcal{f}$ 1947;35:584-602.

19 Pringle TH, Scobie IN, Murray RG, Kesson CM Maccuish AC. Prolongation of the QT interval during therapeutic starvation: a substrate for malignant arrhyththerapeutic starvation: a substrate

20 Rasmussen LH, Andersen T. The relationship between QTc changes and nutrition during weight loss after gastroplasty. Acta Med Scand 1985;217:271-5.

21 Chambers JB, Walto RT, Coupe MO, Ward DE. QT prolongation after ileojejunal bypass. Lancet 1985;ii: 1308

22 Isner JM, Sours HE, Paris AL, Ferrans VJ, Roberts WC Sudden, unexpected death in avid dieters using the liquid-protein-modified-fast diet. Circulation 1979;60 1401-12.

23 Frisoni M, Filipponi C, Gullo I, Poggi VM, Gasbarrini G. Investigation of $\mathrm{QT}$ interval in adult coeliac disease. BMF 1992;304:1285.

24 Jackmann WM, Friday KJ, Anderson JL, Aliot EM, Clark $M$, Lazzara $R$. The long QT syndromes: a critical review, new clinical observations and a unifying hypothesis. Prog Cardiovasc Dis 1988;31:115-72.

25 Day CP, McComb JM, Campbell RWF. QT dispersion: an indication of arrhythmia risk in patients with long $\mathrm{QT}$ intervals. Br Heart $f$ 1990;63:342-5.

26 Aitcheson JD, Higham PD, Furniss SS, Campbell RWF Admission QT dispersion does not predict ventricular fibrillation following acute myocardial infarction. $B r$ Heart 7 1993;69:64.

27 Reynolds T, Martin H, Homann R. Serum electrolytes and the electrocardiogram. Am Heart $\mathcal{f} 1951 ; 42: 671-81$.

28 Loeb HS, Pietras RI, Gunnar RM, Tobin JR. Paroxysmal ventricular fibrillation in two patients with hypomagventricular fibrillation in two patien

29 Hollister RM, Goodwin JF. The electrocardiogram in cardiomyopathy. Br Heart $\mathcal{f}$ 1963;25:357-74.

30 Ahnve S. QT interval prolongation in acute myocardial Ahnve S. QT interval prolongation in acute my

31 O'Donnell J, Lovelace DE, Knoebel SB, McHenry PL. Behaviour of the terminal $T$ wave during exercise in normal subjects, patients with symptomatic coronary disease and apparently healthy subjects with abnormal S
segment depression. $\mathcal{f} \mathrm{Am}$ Coll Cardiol 1985;5:78-84. 